\title{
"Blockchain Plus Supply Chain Finance" Boosts Pharmaceutical Industry: A Case Study of Jixiangtian Blockchain Medical and Health Service Platform
}

\author{
Kong Yan ${ }^{1, a}$, Wang Yanna ${ }^{1, b^{*}}$ \\ ${ }^{1}$ School of Economics and Trade, Guangzhou Xinhua University, Guangzhou, China
}

\begin{abstract}
China's pharmaceutical industry has enormous potential. With the launch of relevant state policies and the innovations being made on the financial front, the country's medical industry has a closer tie with its financial institutions in the supply chain. On the other hand, the booming of supply chain finance provides excellent platforms for financial institutions, pharmaceutical companies and third-part logistics to interact with each other. Nevertheless, supply chain finance in a traditional sense is still facing many challenges. Taking Jixiangtian, a blockchain and medical service platform as an example, this paper illustrated the problems faced by the traditional pharmaceutical supply chain, and analyzed pharmaceutical supply chain platforms using blockchain technology to propose solutions to tackle the financial challenges faced by traditional supply chains.
\end{abstract}

\section{Definition}

\subsection{Supply chain finance}

In the past, supply chain finance (SCF) referred to the services provided by banks to their customers (major enterprises), such as financing, settlements and wealth management, timely loan collection for the suppliers of their customers, as well as payment and inventory financing services. In the modern business world, supply chain finance has a broader definition. Core enterprises working with their upstream and downstream partners, no matter by themselves or through the services of financial institutions, fall under the category of SCF as long as financial products and services are involved in this process.

SCF was born with its major role to integrate multiple financial services, such as financing, wealth management and loan into one chain ${ }^{[1]}$. What lies at the center is the bank supply chain, which circles around the core enterprises and manages the capital flows and logistics of upstream and downstream smaller enterprises. Besides, through risk diversification, under SCF, individual enterprises can put their eggs in more than one basket, turning risks into controllable ones that can be shared by the entire supply chain. Currently, mainstream supply chains in China, such as the bank supply chain, have entered a stage of stable development. What contributed to it is the booming of online finance. Due to the interconnected, easy to operate and timesaving characteristics of the Internet, online financial services will become an inevitable trend for supply chain finance in the future. In addition to the developing online model, SCF also has other advantages like integrating resources across different platforms and ensuring the participants' benefits in the entire supply chain. Based on the SCF service standards, not all enterprises or financial organizations are qualified to become participants in a supply chain. Only those who provide financial services to more than one participant in this supply chain would be considered as service providers in SCF. Such rules can protect the rights of the participants and ensure that each of them can benefit from SCF in an equitable way.

Therefore, when we mention SCF, we generally mean the capital flows under SCF, the entire transaction process and the parties involved in the transaction. Having been restricted for a long time, the rising of SCF rode the wave of the booming Internet technology and economic globalization. Hopefully, breakthroughs can be achieved in today's information era. By presenting the introduction and advantages of SCF, we can figure out SCF's great value to the financial community. Moreover, judging from the current situation inside the industry, SCF's superiority has received wide recognition from nearly all sides. In the future, the SCF's advocates need to work on the system's safety and antirisk issues in order to dispel the concerns of banks and enterprises over this new thing.

\subsection{Blockchain}

Blockchain is a new type of application for computing technologies such as distributed data storage, point-topoint transmission, consensus mechanisms, and encryption algorithms. Essentially a blockchain is a

\footnotetext{
*Corresponding author: 859008061@qq.com
} 
decentralized database. As the underlying technology for Bitcoin, blockchain refers to a series of data blocks that are linked to each other through encryption algorithms. Each data block contains information of Bitcoin Internet transactions of the same batch, used to validate the information (or for anti-counterfeiting) and generate the next block.

\section{Advantages of the Blockchain Technology in Supply Chain Finance}

Blockchain technology renders information immutable. In blockchain technology, the transaction information is sealed with asymmetric encryption. The encryption method requires a huge amount of data operation for decryption, so it can prevent the information from being tampered with, raise the transaction's security level of and ultimately ensure the fairness and legitimacy of the transaction. Tamper-resistant information means when the transaction services are provided by SCF, both parties to the transaction as well as the third party, the Internet platform, are not allowed to change the information, hence preventing illegal acts that tamper with the transaction information by exploiting network loopholes to gain unlawful benefits. Tamper-resistance also means the information cannot be destroyed. Hence illegal acts like unlawful transactions and account cancel exploiting the time gap of fund arrival will be eliminated. In this way, transactions through SCF are more likely to be successful. Hopefully, there will be fewer breaches of contract and frauds during the transaction process. Besides, the untampered information ensures that the information is authentic, and the indestructible property of the information ensures that the entire transaction process can be well-preserved. In this way, the regulator and both parties to the transaction are able to monitor the transaction with the information and maintain a legal transaction environment ${ }^{[2]}$.

The decentralized distributed management helps secure information safety. Due to the use of distributed computing and storage structures, blockchain technology has no centralized features. When used for data processing, information transmission, system maintenance, among others, obvious distributed management will be seen in blockchain technology. Therefore, in a decentralized distributed management system, each node owns equal rights and obligations, which can help prevent severe problems such as system collapses or massive information leakage. The absence of a definite center, on the other hand, means each node can be seen as the center of the entire process. In this case, when one node gets destroyed, irrevocable impacts will not fall on other nodes. Therefore, enterprises participating in SCF only need to be responsible for their own nodes. It also means that they can only enjoy the services and obtain the public information of their own respective nodes. The decentralized distributed management protects the safety of the entire system by ensuring that the destruction of a single node will not put the system in danger, hence improving SCF's overall safety performance. The low-security level will not be a weakness for SCF anymore.

Information openness makes transparent transactions. All nodes in a blockchain are open for viewing. In other words, the content of every node can be viewed at any time, significantly reducing the cost of information acquisition. For participants to a transaction, every move they make leaves a trail. Transparency elevates for each transaction. Moreover, the information up for viewing is tamper-resistant, which ensures the fairness, impartiality and openness of transactions from the roots. Every time a new transaction is conducted, another block will automatically be added downstream on the blockchain to record the information for this transaction. Therefore, all transactions involving an entity will be recorded on the platform. When dealing with others, if we want to know whether there are risks involved, or if we want to calculate the success rate of the transaction, we only need to analyze the transaction records of the other party. Transaction information transparency is very popular in nowadays financial industry. It provides clients with references to decide whether to proceed with the transaction and effectively reduces the risks they need to bear during the transaction.

\section{Case Study}

\subsection{Background}

The problem exists in the traditional pharmaceutical SCF. The industrial chain and the supply chain are similar and closely related to each other. The biggest difference between the two is that the industrial chain does not have a prominent core enterprise, while businesses on the supply chain all revolve around the core enterprises. In a pharmaceutical industry supply chain (Figure 1), the core enterprise is the manufacturer of pharmaceutical products, or pharmaceutical processing companies ${ }^{[3]}$.

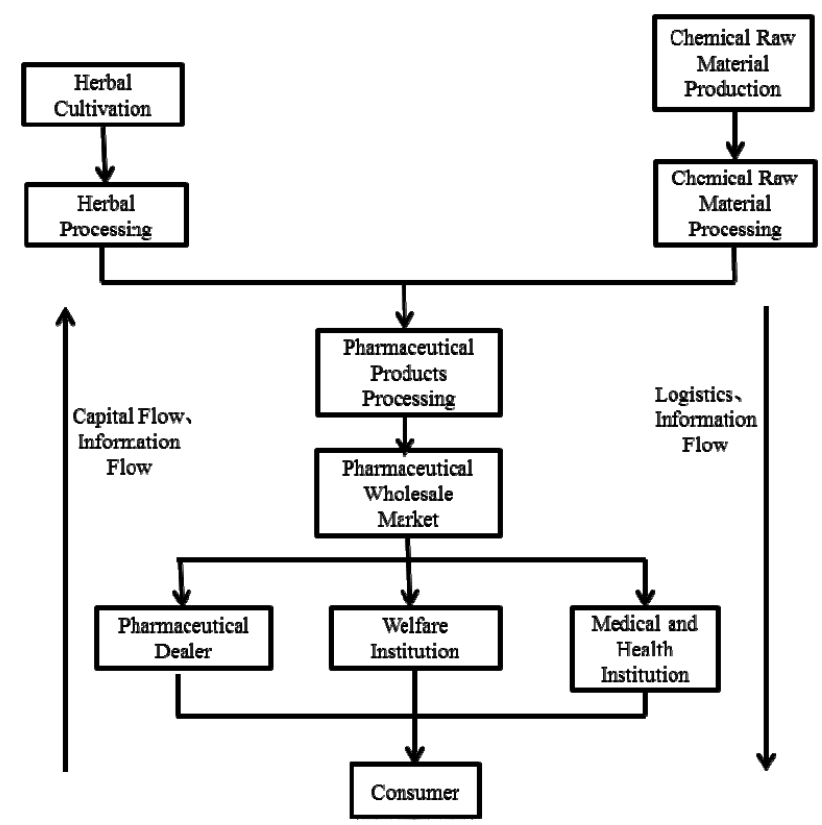

Fig. 1. Supply Chain of Pharmaceutical Industry 
The massive gaps between upstream and downstream enterprises. In the industrial supply chain management of medicine and drug manufacturers, there are various types of enterprises along the chain, ranging from upstream and downstream enterprises. At the same time, various departments inside enterprises on a supply chain need to be coordinated with each other. The lack of smooth and timely information flow increases the management costs. With globalized production and deepening division of labor, upstream enterprises in the industrial chain, such as herbal medicine planting and processing enterprises, chemical raw materials production and processing enterprises, and electronic components production and processing enterprises, tend to choose different geographical locations. Whereas downstream enterprises such as pharmaceutical dealers, social welfare institutions, and medical and health organizations are distributed across the nation or even around the globe. Therefore, it is quite difficult for the core enterprises to monitor the flows of capital, material and information along the industrial chain.

The safety issue of drugs. In the traditional pharmaceutical supply chain, there is not enough transparency during the production process and logistics. Information is absent about the value, production information and data of pharmaceutical products. This phenomenon leads to the drug safety issue. If commercial banks without this information lend money to these enterprises, there is a possibility that harmful or counterfeit drugs will find their way into the market. But on the other hand, it is very difficult for us to pinpoint the source of these unqualified products.

The issue of warehouse supervision. There are many types of pharmaceutical products. Different types of drugs require different ways of storage and transportation. For instance, some liquid medicines have temperature requirements, some should be protected from sunlight and some with a specified period for clinical use. No specific data exists in terms of what these drugs really need, which poses challenges to warehousing and transportation. Therefore, problems like inventory turnover and collateral risks exist under the traditional SCF.

The issues with the credit system and financing. Due to the absence of data that can demonstrate the credibility of upstream and downstream enterprises, the financing costs are relatively high for upstream and downstream enterprises, especially those at the third- or fourth-tier small or medium-sized suppliers and distributors far away from the core enterprise. From the perspective of risk control, most banks are only willing to offer loans to first-tier suppliers and distributors. The result is that enterprises at both ends of the supply chain will not get enough loans. They tend to reduce the supply and purchase of pharmaceutical products, which in turn lead to a decline in the service quality of the entire supply chain, compromising the interests of all enterprises along the chain ${ }^{[4]}$.

\subsection{Introduction}

Jixiangtian is a blockchain medical and health service platform as a result of the strategic partnership between Shandong Realcan Pharmaceutical Group and China Zheshang Bank. Based on Internet technologies such as blockchain, big data and artificial intelligence (AI), Jixiangtian aims to provide services for the core enterprises and their upstream and downstream partners along the industrial chains, and provide solutions for the financing problems of SMEs with the ultimate goal of promoting the nation's SCF and financial inclusion strategy.

\subsection{Case Study}

Jixiangtian is the first blockchain medical and health service platform jointly built by a pharmaceutical enterprise and a financial institution inside the industry. This platform will integrate Zheshang Bank's leading blockchain technology and financial services innovation capabilities based on Ruixin, a pharmaceutical SCF financial business platform that Realcan has built for several years. Its aim is to create a pharmaceutical business data platform covering upstream pharmaceutical suppliers and distributors, medical institutions and financial institutions. It also aims to provide information and financial connectivity for the entire medical and health industrial chain and create a new ecological model for the industry (Figure 2).

Jixiangtian gathers, transmits and detects the financing needs of various entities along the industrial chain by utilizing Realcan's SCF platform and providing underlying authentic business data relevant to those financing needs. Through risk management and credit infusion, the financing needs and risk control data can be quickly and directly transmitted to the funders, opening up the financing channels of the entire industrial chain, and ultimately raising the values created along the industrial chain. Based on its industry-leading SAP information platform, Realcan Pharmaceutical has created another innovative product with financial technology: "Ruixin" (e-payment commitment letter that can be split, transferred and supports financing). Ruixin helps realize the online management of supply chain trades and later developed into Realcan's SCF platform. The launch of Jixiangtian has offered solutions to financial problems that existed in the traditional pharmaceutical supply chain. 


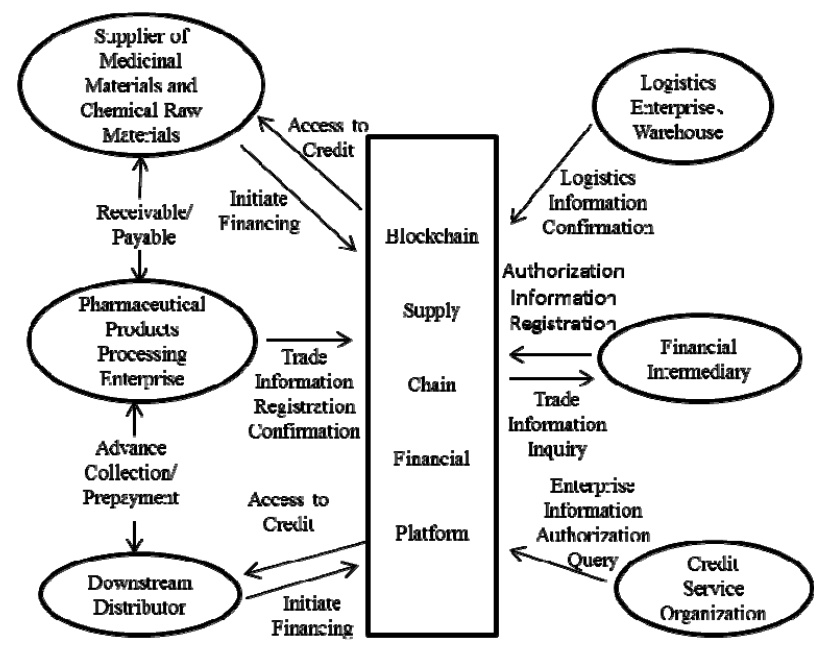

Fig. 2. Blockchain Supply Chain Financial Platform

First, the massive gaps between upstream and downstream enterprises in the supply chain was narrowed. The pharmaceutical supply chain loaded with blockchain technology offered solutions to the delayed and problematic information transmission due to scattered geographical locations. Any records of material, capital and information flows were kept in the blockchain. Pharmaceutical product manufacturers can real-time monitor the supply chain to effectively manage all enterprises along the supply chain in a targeted manner.

Second, the safety issue of drugs was tackled. The safety of medical supplies is always our top concern during production. In a blockchain, each block recorded the value and production information of the medical supplies, and that information was tamper-resistant. So before granting loans to these enterprises, banks can decide whether this is a bad pharmaceutical supply chain based on this information, which can not only reduce counterfeit drugs in the market, but also lower the risk of bad loans.

Third, problems with warehouse logistics were solved. The blockchain recorded information related to every link ranging from production and sales, as well as information about the storage and quantity requirements of drugs. Pharmaceuticals with the same storage requirements that will not react with each other can be stored in the same warehouse and transported in the same logistics means, lowering the marginal costs of storage and logistics and elevate the inventory turnover of supply chain enterprises.

Fourth, problems with the credit system and financing no longer exist. Due to the tamper-proof and transparency of blockchain technology, data recorded in the blockchain are highly reliable. Some SMEs in the supply chain suffering from financing difficulties due to the lack of a credit evaluation system found proof of their reliability from the records kept by the blockchain. The SMEs along a supply chain have to pay lower financing costs, but the number of loans they get keeps rising, hence improving the service quality of the entire pharmaceutical supply chain.
Fifth, problems with financing tool transfer were addressed. Blockchain technology is used to issue a new type of digital invoice that can be split and transferred openly and transparently, so that the credit system of the entire supply chain will become traceable and referable. This can provide opportunities for enterprises with financing difficulties, lowering their financing costs and for the banks, reduce the risks of bad loans.

\section{Conclusions}

(1) The consensus mechanism and decentralized feature ensure that the data on the blockchain will not be tampered with or fabricated.

(2) SCF contributes to the sustainable operation of the core enterprises and their upstream and downstream partners.

(3) SCF is the best application scenario for blockchain technology.

(4) The booming of blockchain technology drives reforms in financial risk control. China's SCF has entered a 4.0 period.

\section{Acknowledgment}

The authors are grateful for the financial support of the Youth Innovation Project of Key Platform of Colleges and Universities in Guangdong Province (2016WQNCX188); and the Project of Teaching Team of Internet Finance Course (2019T002) in Xinhua College of Sun Yat-sen University; the 2016 Department of Education of Guangdong Province Key Discipline "Public Administration" research project;

\section{References}

1. Liang L.Y. Analysis on the development of supply chain finance based on blockchain technology -Taking the pharmaceutical industry as an example [J]. China Journal of Commerce, 2018(25):7-8.

2. Wang Y., Yu L.J. Research on the financial development of supply chain based on blockchain technology $[\mathrm{J}]$. China Circulation Economy, 2019(29):162-163.

3. Lu J.Y. Analysis of supply chain finance solutions for pharmaceutical distribution enterprises [J]. Times Finance, 2019(29):114-116.

4. Gao C.C. Review and Prospect of supply chain finance in the field of medicine circulation [J]. Economic Research Guide, 2020(23):103-105. 